\title{
Évaluation de la couverture vaccinale antigrippale du personnel du pôle de médecine d'urgence du centre hospitalo-universitaire de Toulouse lors de la saison hivernale 2017-2018
}

\author{
Evaluation of the Anti-influenza Vaccination Coverage of the Staff of the Emergency Medicine \\ Center of Toulouse University Hospital Center during the Winter Season 2017-2018
}

\author{
N. Vialaret Du Val De La Croix · M. Oberlin · E. Dehours $\cdot$ S. Charpentier \\ Reçu le 6 novembre 2018; accepté le 16 janvier 2019 \\ (C) SFMU et Lavoisier SAS 2019
}

Résumé Introduction : Plus de 677000 patients ont consulté de décembre 2016 à avril 2017 en structure des urgences (SU) en Occitanie. La proportion de patients à risque de grippe grave est plus importante en SU que dans la population générale. Les professionnels de santé doivent être vaccinés pour lutter contre la propagation du virus ainsi que pour protéger les patients. L'objectif de notre étude était de connaître le taux de vaccination antigrippale parmi les professionnels de santé travaillant dans le pôle de médecine d'urgences (PMU) d'un centre hospitalier universitaire (CHU) durant l'hiver 2017-2018.

Matériel et méthode : Nous avons réalisé une étude épidémiologique rétrospective monocentrique sur le PMU. Les critères d'inclusion étaient les professionnels de santé en poste (médecin, infirmier, interne ou aide-soignant) dans les SU de Purpan, Rangueil et/ou au service d'aide médicale urgente de Haute-Garonne (Samu 31) entre le 30 novembre 2017 et le 30 avril 2018. Le critère de jugement principal était défini par la proportion de professionnels de santé vaccinés. Les critères de jugement secondaires étaient la recherche de facteurs motivant la vaccination ou la non-

N. Vialaret Du Val De La Croix $(\bowtie) \cdot$ E. Dehours

Structure d'urgences, centre hospitalier universitaire

Toulouse-Purpan, place du Docteur-Joseph-Baylac,

F-31300 Toulouse, France

e-mail : nicolasvialaret@gmail.com

M. Oberlin

Structure d'urgences, centre hospitalier Jean-Rougier,

335, rue Wilson, F-46000 Cahors, France

S. Charpentier

Service des urgences, centre hospitalier universitaire de Toulouse,

9, place Lange, 60033, F-31059 Toulouse, France

Équipe 5, Inserm UMR 1027, 37,

université Toulouse-III-Paul-Sabatier

allées Jules Guesde, F-31000 Toulouse, France vaccination. Les résultats étaient exprimés en pourcentages avec calcul de l'intervalle de confiance à $95 \%$.

Résultats : Deux cent trente-six professionnels de santé (59\%) ont répondu au questionnaire, 103 (44\%) étaient vaccinés contre la grippe. Les facteurs évoqués pour la vaccination étaient la protection des patients, se protéger soimême et son entourage. L'absence d'envie, l'impression de non-efficacité du vaccin, la peur des effets secondaires et la méconnaissance des principes de la vaccination étaient les facteurs retrouvés pour la non-vaccination.

Conclusion : Le taux de vaccination du personnel du PMU du CHU reste faible vis-à-vis des objectifs fixés par Santé publique France qui sont de 75 \%. La mise en place d'un professionnel référent dans le service qui effectuerait une vaccination après information et sensibilisation est une piste à évaluer.

Mots clés Grippe · Urgence · Professionnel de santé · Vaccination

Abstract Introduction: More than 677,000 patients consulted from December 2016 to April 2017 in emergency department (ED) in Occitanie. The proportion of patients at risk for severe influenza is higher in ED than in the general population. The healthcare workers must be vaccinated to fight the spread of the virus as well as to protect patients. The objective of our study was to know the influenza vaccination rate among the healthcare workers working at the Emergency Medicine Center (EMC) of the University Hospital Center (CHU) during the winter of 2017-2018.

Methods: We realized a retrospective monocentric epidemiological study on the EMC. The inclusion criteria were the healthcare workers in position (physicians, nurse, intern, or caregiver) in the ED of Purpan, Rangueil, and/or emergency medical help service (Samu) of the Haute-Garonne (Samu 31) from November 30, 2017 to April 30, 2018. 
The primary endpoint was defined by the proportion of immunized healthcare workers. The secondary endpoint was to research the motivation for the vaccination or the non-vaccination. The results were expressed as percentages with calculation of the $95 \%$ confidence interval.

Results: Two hundred and thirty-six (59\%) health professionals responded, and 103 (44\%) were vaccinated against influenza. The factors mentioned for the vaccination were the protection of the patients, to protect oneself and one's entourage. The absence of envy, the impression of noneffectiveness of the vaccine, the fear of side effects, and the ignorance of the principles of vaccination were the factors found for the non-vaccination.

Conclusion: The vaccination rate of the staff of the EMC of the University Hospital Center remains low compared to the objectives set by Santé Publique France which are 75\%. The establishment of a referral professional in the service who would carry out a vaccination after information and awareness is a track to evaluate.

Keywords Influenza Emergency $\cdot$ Healthcare workers · Vaccine

\section{Introduction}

Plus de 2,4 millions de patients ont consulté pour syndrome grippal durant la saison hivernale 2017-2018 en France, dont 75467 passages en structure des urgences (SU). Le taux d'hospitalisation a été particulièrement élevé : 9738 hospitalisations contre 6500 en 2016-2017 et 3400 en 20152016 [1]. Le nombre de cas graves admis en réanimation est le plus élevé depuis la saison 2009-2010 (2 915 cas). Parmi ceux-ci, $56 \%$ n'étaient pas vaccinés [1]. Au moins 17900 décès ont été constatés de novembre 2017 à fin avril 2018 concernant majoritairement des personnes de 65 ans et plus [1]. Plus de 1,8 million de patients ont consulté une SU dans la région Occitanie lors de l'année 2017 (+ $3 \%$ par rapport à 2016) [2,3] et plus de 677000 lors de la période d'épidémie grippale (de novembre 2016 à avril 2017), dont 3700 pour « syndrome grippal » et 8200 pour « fièvre ». Deux cent trente-sept patients ont consulté dans une SU du centre hospitalier universitaire (CHU) de Toulouse pour « syndrome grippal » et 253 pour « fièvre » pour la même période (source ORU-MiP).

Le personnel du pôle de médecine d'urgences (PMU) travaillant en SU ou en service d'aide médicale urgente (Samu) est en contact permanent avec des patients fragiles et des patients grippés. D'après les études internationales, $42 \%$ des patients pris en charge en SU sont à risque de grippe grave contre $31 \%$ dans la population générale [4-6]. Il est donc nécessaire que le personnel soignant du PMU soit vac- ciné pour lutter contre la propagation du virus et pour protéger les patients à risque. Le personnel hospitalier médical et paramédical est un des acteurs de soins dans la lutte contre la propagation de la grippe saisonnière. La vaccination globale des soignants permet une diminution de la mortalité jusqu'à $20 \%$ au niveau des patients institutionnalisés [7]. Elle permet de plus une diminution de l'absentéisme au travail et une baisse de la fréquence des infections grippales au travail [7]. Cependant, la vaccination du personnel soignant hospitalier reste faible par rapport à la population générale. Elle est de $27 \%$ d'après les dernières études de Santé publique France $[8,9]$ alors que l'objectif fixé est de $75 \%$. La vaccination est recommandée pour le personnel de santé intrahospitalier mais reste non obligatoire [10]. L'objectif de notre étude était de connaître le taux de vaccination antigrippale parmi les professionnels de santé travaillant dans le PMU du CHU de Toulouse qui comprend les SU de Purpan, Rangueil et le Samu de la Haute-Garonne (Samu 31) durant la saison 2017-2018.

\section{Matériel et méthode}

Nous avons réalisé une étude épidémiologique rétrospective monocentrique au sein du PMU du CHU de Toulouse (SU de Rangueil et Purpan, Samu 31) durant la saison hivernale 2017-2018 (novembre 2017 à avril 2018). L'objectif principal était de connaître le taux de vaccination antigrippale parmi les professionnels de santé travaillant dans le PMU du CHU de Toulouse. L'objectif secondaire était de connaître les facteurs évoqués pour la vaccination et la nonvaccination par les professionnels interrogés.

Le critère de jugement principal était défini par le nombre de personnes ayant une couverture vaccinale antigrippale durant la saison 2017-2018 parmi les professionnels travaillant dans le PMU du CHU de Toulouse. Les critères de jugement secondaires étaient les facteurs évoqués par les professionnels vaccinés et non vaccinés.

Les professionnels de santé du PMU du CHU de Toulouse (SU de Purpan, Rangueil et Samu 31) comprenant les médecins, infirmiers, internes et aides-soignants (AS) étaient inclus dans l'étude. Tout personnel hors PMU du CHU de Toulouse et les étudiants en médecine (externes), les ambulanciers, les assistants de régulation médicale étaient exclus. La liste exhaustive des soignants infirmiers diplômés d'État (IDE) et AS travaillant sur le PMU du CHU de Toulouse était recueillie auprès des cadres de santé hospitaliers des trois services. Les secrétariats médicaux des SU de Purpan et Rangueil ont fourni la liste des médecins et internes en poste sur la période. La liste des médecins en poste au Samu 31 était recueillie auprès du secrétariat du Samu 31. Un questionnaire a été envoyé par mail aux adresses professionnelles de chacun. Des questionnaires papiers ont été 
distribués au sein du PMU. Le questionnaire figure en annexe. La première partie comprenait la collecte de données personnelles telles que l'âge, le sexe et la profession. La deuxième partie du questionnaire interrogeait sur le choix de la vaccination ou non, les antécédents de grippe et de vaccination antigrippale les années précédentes. Pour les personnes vaccinées, le moyen de vaccination était demandé. La troisième partie du questionnaire recherchait les facteurs évoqués pour la vaccination ou la nonvaccination.

\section{Analyse statistique}

L'analyse des données anonymisées a été réalisée à l'aide des logiciels Word ${ }^{\circledR}$, Excel ${ }^{\circledR}$ (Microsoft, Redmond, ÉtatsUnis, version 2016). Toutes les variables de l'étude ont été analysées de façon individuelle avec vérification du nombre de données manquantes et de valeurs aberrantes. La normalité de chaque distribution pour les variables quantitatives a été analysée. Les questionnaires ont été remplis de manière exhaustive par les personnels interrogés, la proportion de données manquantes était négligeable, et il n'a pas été utilisé de méthode d'imputation pour les valeurs manquantes. Les mesures de dispersion de chacune de ces variables ont été évaluées en fonction de la normalité : la distribution des variables quantitatives est représentée par la médiane et l'intervalle interquartile si la distribution n'est pas normale (médiane [p25\%-p75]), elle est représentée par la moyenne suivie de l'écart-type si la distribution est normale. Les données qualitatives sont exprimées en nombre et pourcentage. Les analyses comparatives ont été réalisées en utilisant le test $\mathrm{du} \mathrm{Chi}^{2}$ de Pearson pour les comparaisons de pourcentages et le test du $t$ de Student pour les comparaisons de moyennes.

Le seuil de significativité statistique est considéré atteint quand le risque d'erreur est inférieur à $5 \%(p<0,05)$. Pour une hypothèse d'une couverture vaccinale à $45 \%$ (taux de vaccination de la population générale) avec une IC $95 \%$ pour une précision de plus ou moins $10 \%$, le nombre de sujets nécessaires était de 95 vaccinés, soit 211 sujets.

\section{Résultats}

Au total, 236 (59\%) professionnels de santé ont répondu au questionnaire du 30 novembre 2017 au 30 avril 2018 (77 AS, 193 IDE, 43 internes, 85 médecins). Cent trois professionnels de santé (44\%) ont été vaccinés. Le taux de vaccination pour chaque catégorie professionnelle est représenté dans le tableau 1. La moyenne d'âge était identique chez les non-vaccinés et chez les vaccinés ( $32 \pm 9$ ans). Les personnels de plus de 50 ans ne sont pas significativement plus vaccinés que les personnels de moins de 50 ans ( 47 vs $43 \%, p>0,05$ ). Les hommes représentaient $32 \%$ de notre population et étaient significativement plus vaccinés que les femmes ( 39 vs $26 \%, p=0,015$ ).

Parmi le personnel vacciné cette saison, 17 (16\%) n'ont jamais été vaccinés (sept IDE, deux AS, six internes, deux médecins). Parmi le personnel non vacciné, 105 (79\%) n'ont jamais été vaccinés. Parmi le personnel interrogé ayant déjà eu la grippe $(131$ [56 \%]), $72(55 \%)$ n'ont pas été vaccinés cette année. Si la vaccination a été réalisée, 51 (49\%) ont été vaccinés à la médecine du travail, sept (7\%) en cabinet de médecine générale et 45 (44\%) par leurs propres moyens. Les facteurs évoqués pour la réalisation ou la non-réalisation de la vaccination sont résumés dans les tableaux 2, 3. Le taux de vaccination était significativement différent entre interne-médecin et IDE-AS $(p<0,001)$. Il n'existait pas de différence significative entre AS et IDE et entre médecins et internes.

\section{Discussion}

Dans notre étude, $44 \%$ des professionnels de santé du PMU du CHU de Toulouse étaient vaccinés contre la grippe lors de la saison hivernale 2017-2018. Ce chiffre reste en dessous de l'objectif de $75 \%$ fixé par Santé publique France. Cependant, il reste plus élevé que dans certaines études nationales ou internationales où le taux de vaccination ne dépasse pas $20 \%[11,12]$. À titre de comparaison, dans la population globale, la couverture vaccinale tous régimes est estimée à

\begin{tabular}{|c|c|c|c|}
\hline Profession & Nombre d'agents interrogés, $n(\%)$ & Taux de vaccination, $n(\%)$ & $\begin{array}{l}\text { IC } 95 \% \text { du taux } \\
\text { de vaccination }\end{array}$ \\
\hline AS & 35/77 (46) & $9(26)$ & $11-40$ \\
\hline IDE & 112/193 (58) & $19(17)$ & $10-24$ \\
\hline Interne & 39/43 (91) & $37(95)$ & $88-100$ \\
\hline Médecin & $50 / 85(59)$ & $37(78)$ & $67-90$ \\
\hline
\end{tabular}


Tableau 2 Facteurs évoqués lors de l'absence de vaccination

$n(\%) \quad$ IC $95 \%$

«Le vaccin est non efficace »

« Non efficace à $100 \%$, car il existe de nombreuses souches différentes »

57 (43) $34-51$

«J'ai été atteint de la grippe l'année où je me suis fait vacciner»

13 (10) $5-15$

« Il existe des effets secondaires du vaccin »

« Le vaccin peut donner la grippe»

18 (13) $8-19$

«Le vaccin peut entraîner des effets secondaires non connus liés aux substances contenues dans le vaccin »

$40(30) \quad 22-38$

«Je n'ai pas besoin du vaccin »

« Je ne suis jamais malade »

26 (19) $13-26$

« Je suis immunisé grâce aux contacts fréquents aux urgences »

13 (10) $5-15$

« je ne fais pas partie de la population à risque»

23 (17) $11-24$

« Mon système immunitaire est efficace et je suis protégé contre la grippe »

8 (6) $\quad 2-10$

« La grippe est une pathologie bénigne pour mon âge et mon terrain »

20 (15) $9-21$

« Je n'ai tout simplement pas envie de faire le vaccin »

76 (58) 49-66

«J'ai des difficultés pour trouver un moment pour la vaccination »

« Mes horaires de travail entraînent la vaccination difficile »

13 (10) $5-15$

« Les horaires de la médecine du travail entraînent des difficultés pour y aller aux vues de mes propres horaires »

« J'oublie constamment de me faire vacciner»

21 (16) $10-22$

«Idées personnelles»

«Il n'a pas prouvé son efficacité »

19 (14) $8-20$

« Je ne fais aucun vaccin non obligatoire »

31 (23) $16-30$

« Commercial, sert à enrichir les industries pharmaceutiques »

$n:$ nombre

Les résultats sont exprimés en nombre (\%) et intervalles de confiance à $95 \%$ (IC $95 \%$ ) du pourcentage

Tableau 3 Facteurs évoqués lors de la réalisation de la vaccination

\begin{tabular}{|lll|}
\hline & $n(\%)$ & IC \\
& $95 \%$ \\
\hline Pour protéger les patients & $82(80)$ & $72-88$ \\
Pour protéger l'entourage & $70(68)$ & $59-77$ \\
Pour me protéger & $77(75)$ & $67-83$ \\
Parce que c'est le devoir de tout soignant & $35(34)$ & $25-43$ \\
Parce que j'ai été sensibilisé & $12(12)$ & $5-18$ \\
par les campagnes de sensibilisation & \\
à l'hôpital & \\
Parce qu'on me l'a recommandé & \\
\hline$n:$ nombre & $16-32$ \\
\hline $\begin{array}{l}\text { Les résultats sont exprimés en nombre (\%) et intervalles de } \\
\text { confiance à 95 \% (IC 95 \%) du pourcentage }\end{array}$ \\
\hline
\end{tabular}

$46 \%$, stable par rapport à la saison 2016-2017 (46 \% au 28 février 2017). Elle est estimée à $50 \%$ chez les 65 ans et plus, et à $29 \%$ chez les personnes à risque de moins de 65 ans [1].
Le taux de vaccination est très différent en fonction de la profession des personnes interrogées. Les médecins et les internes sont significativement plus vaccinés que les AS et les IDE ( $p<0,001$ pour toutes les comparaisons). Cette différence est retrouvée dans de nombreuses études [11-15] où le taux de vaccination allait de 50 à $86 \%$ pour les médecins, alors qu'il était de 11 à $55 \%$ pour les IDE et AS dans les SU ou dans les centres hospitaliers tous services confondus. Le pourcentage d'internes vaccinés est très élevé. Ce résultat est très positif pour l'avenir. Les futurs médecins sont convaincus par la vaccination et pourraient être un relais efficace pour sensibiliser les professionnels hésitants.

Dans notre étude, nous ne retrouvons pas d'association entre vaccination et âge. Certaines études [13,16,17] suggèrent qu'un âge de plus de 50 ans serait associé à un meilleur taux de vaccination chez les professionnels. Cette différence n'est pas retrouvée dans notre étude. Cette absence d'association entre âge et vaccination est probablement liée à notre échantillon qui comprend des médecins jeunes (internes et assistants). Les études retrouvant une association entre âge et vaccination des professionnels n'avaient pas analysé les sous-groupes de professionnels. 
Les facteurs évoqués chez les professionnels vaccinés sont la protection des patients, le fait de se protéger soimême ainsi que de protéger son entourage. De manière moins importante, les professionnels de santé se sont vaccinés après recommandation et par devoir. La sensibilisation par les campagnes de l'hôpital a relativement peu d'impact dans notre population vaccinée. Les professionnels motivés pour se vacciner ne sont a priori pas limités par les heures d'ouverture de la médecine du travail ou l'accès au vaccin. Ils se font vacciner essentiellement à la médecine du travail (49\%), et il ne semble pas y avoir de limitation liée aux heures d'ouverture de celle-ci. En seconde intention, les professionnels se vaccinent par leurs propres moyens (essentiellement dans le service où ils travaillent). Ces résultats sont sensiblement similaires aux résultats retrouvés dans la bibliographie. Dans une revue de la littérature de 2009, les motivations principales de la vaccination des professionnels étaient par ordre de fréquence : se protéger soi-même, protéger les patients puis protéger les proches [16]. Une étude réalisée en 2013 au CHU de Lyon retrouvait pour $82 \%$ des vaccinés un objectif de protection des proches, puis de se protéger soi-même $(66 \%)$ et enfin de protéger les patients $(57,1 \%)[13]$.

Concernant les facteurs évoqués chez les professionnels non vaccinés, $58 \%$ ont cité l'item " je n'ai tout simplement pas envie de me faire vacciner ». Cette donnée interpelle quant à l'absence de prise de conscience de la nécessité de vaccination pour la protection des personnes à risque. Le personnel non vacciné semble convaincu que le vaccin n'est pas utile. Parmi eux, $79 \%$ n'ont jamais été vaccinés. Cette donnée illustre la difficulté de vacciner le personnel ne s'étant jamais fait vacciner. Les autres facteurs évoqués tiennent d'une méconnaissance des principes de la vaccination antigrippale. Ainsi, plus d'une personne sur trois pense que le vaccin n'est pas efficace et qu'il existerait trop de souches différentes pour que celui-ci soit efficace. Plus d'une personne sur six pense que se faire vacciner peut donner la grippe. Une personne sur trois pense qu'il existe des effets secondaires non connus. Une personne sur cinq pense ne jamais être malade, que le vaccin est une pathologie bénigne pour leur âge et qu'ils ne font pas partie de la population à risque. Ces résultats sont retrouvés au niveau d'une étude lyonnaise publiée en 2013 [13]. Les motivations évoquées chez les professionnels non vaccinés étaient que la grippe est considérée comme une pathologie bénigne (52\%) et plus de la moitié des soignants se déclarent contre la vaccination. Dans une revue de la littérature internationale publiée en 2009 [16], les facteurs principaux évoqués par les professionnels non vaccinés étaient dans l'ordre décroissant : la peur des effets secondaires du vaccin, le manque d'intérêt vis-à-vis du vaccin, le manque de disponibilité et le doute sur l'efficacité du vaccin. Il semblerait que l'accès au vaccin ne soit pas un facteur limitant de la vaccination. Moins d'une personne sur dix a répondu à l'item « mes horaires de travail entrainent la vaccination difficile » et moins de $16 \%$ ont répondu à l'item "les horaires de la médecine du travail entraînent des difficultés pour y aller aux vues de mes propres horaires ».

Une originalité de notre service est que certaines personnes du personnel soignant se sont fait vacciner directement dans le service par un médecin urgentiste après entretien personnel. Le vaccin était réalisé par la suite, et aucune personne n'a refusé la vaccination lorsque le vaccin était immédiatement accessible. Cette action peut être une piste pour l'avenir en désignant un médecin ou une IDE référente en charge de réaliser des entretiens personnalisés et de réaliser la vaccination. Des campagnes d'informations pourraient être menées pour informer les professionnels sur les principes de la vaccination antigrippale. Des études complémentaires pourraient être conduites pour comprendre l'absence d'intérêt de la vaccination chez les professionnels de santé et adapter ainsi le message à leur donner.

Il existe des limites à notre étude. Premièrement, les brancardiers intervenant dans la SU n'ont pas été inclus dans notre étude, car ils appartiennent à un pôle différent. Deuxièmement, nous ne connaissons pas l'impact de cette vaccination sur le nombre d'épisodes de grippe parmi le personnel soignant (grippe déclarée ou arrêt de travail).

\section{Conclusion}

Le taux de vaccination des professionnels de santé du PMU du CHU de Toulouse reste faible vis-à-vis des objectifs fixés par Santé publique France. Il est cependant plus important que dans les études retrouvées dans la bibliographie. Il existe toujours beaucoup d'idées reçues au sein de notre structure avec un impact faible des campagnes de sensibilisation. Il pourrait être intéressant d'évaluer la pertinence d'un médecin référent " intra-muros » réalisant des entretiens individuels permettant une vaccination immédiate dans les suites de l'entretien.

Conflit d'intérêt : les auteurs déclarent ne pas avoir de lien d'intérêt.

\section{Références}

1. Bourdillon F (2018) Grippe. Bulletin hebdomadaire, Santé publique France. http://invs.santepubliquefrance.fr/content/download/146390/533086/version/263/file/Bulletin grippe_S16_bilan preliminaire.pdf (Dernier accès le 17 mai 2018)

2. Équipe ORU (2017) Observatoire régional des urgences. Panorama Occitanie, Activité des structures d'urgence 2016. https:// www.orumip.fr/wpcontent/uploads/2018/07/Panorama2017.pdf (Dernier accès le 19 mai 2018) 
3. Équipe ORU (2016) Observatoire régional des urgences (2016). Panorama Occitanie, Activité des structures d'urgence 2015. https://www.orumip.fr/wpcontent/uploads/2017/11/Panorama2016. pdf (Dernier accès le 19 mai 2018)

4. Loubet P, Samih-Lenzi N, Galtier F, et al (2016) Factors associated with poor outcomes among adults hospitalized for influenza in France: a three-year prospective multicenter study. J Clin Virol 79:68-73

5. Pilly E (2018) Grippe. In: Équipe Collège des universitaires de maladies infectieuses et tropicales. Maladies infectieuses et tropicales $26^{\mathrm{e}}$ édition. ALINEA Plus, Paris, pp 175-82

6. Hiller KM, Sullivan D (2009) Influenza vaccination in the emergency department: are our patients at risk? J Emerg Med 37:439-43

7. Lemaitre M, Meret T, Rothan-Tondeur M, et al (2009) Effect of influenza vaccination of nursing home staff on mortality of residents: a cluster-randomized trial. J Am Geriatr Soc 57:1580-6

8. Vaux S, van Cauteren D, Guthmann JP, et al (2012) Influenza vaccination coverage against seasonal and pandemic influenza and their determinants in France: a cross-sectional survey. BMC Public Health 11:30

9. Guthmann JP, Fonteneau L, Bonmarin I, et al (2012) Influenza vaccination coverage one year after the $\mathrm{A}(\mathrm{H} 1 \mathrm{~N} 1)$ influenza pandemic, France, 2010-2011. Vaccine 30:995-7

10. Bourdillon F (2016) Calendrier des vaccinations et recommandations vaccinales 2016. http://invs.santepubliquefrance.fr/content/ download/124849/443581/version/58/file/beh_calendrier_vaccinations_2016.pdf (Dernier accès le 15 novembre 2018)
11. Hulo S, Nuvoli A, Sobaszek A, et al (2017) Knowledge and attitudes towards influenza vaccination of health care workers in emergency services. Vaccine 35:205-7

12. Zielonka TM, Szymańczak M, Jakubiak J, et al (2016) Influenza vaccination coverage rate for medical staff: influence of hospitalbased vaccination campaign. Adv Exp Med Biol 885:31-8

13. Valour F, Bénet T, Chidiac C, et al (2013) Pandemic A(H1N1) 2009 influenza vaccination in Lyon University Hospitals, France: perception and attitudes of hospital workers. Vaccine 31:592-5

14. Guthmann JP, Fonteneau L, Ciotti C, et al (2011) Couverture vaccinale des soignants travaillant dans les établissements de soins de France. Résultats de l'enquête nationale Vaxisoin, 2009. Bull Epidemiol Hebd 36:371-6

15. Guthmann JP, Abiteboul D (2011) Vaccinations chez les soignants des établissements de soins de France, 2009. Couverture vaccinale, connaissances et perceptions vis-à-vis des vaccinations, rapport final. Institut de veille sanitaire. http://invs.santepubliquefrance.fr/ Publications-et-outils/Rapports-et-syntheses/Maladies-infectieuses/ 2011/Vaccinations-chez-les-soignants-des-etablissements-de-soinsde-France-2009 (Dernier accès le 20 juin 2018)

16. Hollmeyer HG, Hayden F, Poland G, et al (2009) Influenza vaccination of health care workers in hospitals: a review of studies on attitudes and predictors. Vaccine 27:3935-44

17. Saluja I, Theakston KD, Kaczorowski J (2005) Influenza vaccination rate among emergency department personnel: a survey of four teaching hospitals. CJEM 7:17-21 